\title{
Muon and neutrino energy reconstruction for $\mathrm{KM} 3 \mathrm{NeT}$
}

\author{
Evangelia Drakopoulou ${ }^{1,2, a}$ for the KM3NeT Collaboration \\ ${ }^{1}$ N.C.S.R. Demokritos, Patriarchou Grigoriou and Neapoleos, Agia Paraskevi, Greece \\ ${ }^{2}$ National Technical University of Athens, Heroon Polytechniou 9, Zografou Campus, Greece
}

\begin{abstract}
KM3NeT/ARCA is a European deep-sea research infrastructure that will host a neutrino telescope with a volume of several cubic kilometers at the bottom of the Mediterranean Sea. The telescope will search for galactic and extragalactic neutrinos from astrophysical sources like gamma ray bursts, super-novae or colliding stars. The analyses performed in large water Cherenkov detectors rely upon the reconstruction of the muon direction and energy, and consequently, those of the neutrino. The estimation of the muon energy is also critical for the differentiation of muons from neutrinos originating from astrophysical sources from muons and neutrinos that have been generated in the atmosphere and constitute the detector background. The energy is derived from the detection of the Cherenkov light produced by the muons that are created during the charged current interactions of neutrinos in or in the vicinity of the detector. We describe a method to determine the muon and neutrino energy employing a Neural Network. An energy resolution of about 0.29 has been achieved for muons at the $\mathrm{TeV}$ range.
\end{abstract}

\section{Introduction}

One of the aims of the KM3NeT collaboration is to deploy a neutrino telescope of several cubic kilometers at the bottom of the Mediterranean Sea in order to search for neutrinos of galactic and extragalactic origin. This project is called KM3NeT/ARCA. This detector consists of two building blocks of about one hundred detection units that are linked each other and to shore by means of electro-optical cables. Each detection unit hosts 18 optical modules (OMs) vertically aligned on a string. Each optical module consists of a 17 inches glass sphere with 31 photomultipliers (PMTs). These PMTs will detect the Cherenkov light emitted by charged particles which are produced during the neutrino interactions with the Earth or the sea water. The detector background consists of atmospheric $v_{\mu}$ and $\mu$ events which are induced by cosmic rays interactions in the atmosphere and travel through the detector volume leaving similar signatures of astrophysical $v_{\mu}$ and $\mu$. Moreover, photons due to bioluminescence and ${ }^{40} K$ decays in sea water are detected by the PMTs thus originating an optical background.

Two different samples of simulated Charged Current $v_{\mu}$ events were used for this analysis to prevent from adding bias in our results. The first event sample was used to train and test the Neural Network while the second event sample was used during the evaluation procedure. Isotropic atmospheric $v_{\mu}$ flux (by Bartol) [1] was assumed, for both samples, with neutrino

\footnotetext{
ae-mail: liliadrak@inp.demokritos.gr
}

(C) The Authors, published by EDP Sciences. This is an Open Access article distributed under the terms of the Creative Commons Attribution License 4.0 (http://creativecommons.org/licenses/by/4.0/). 


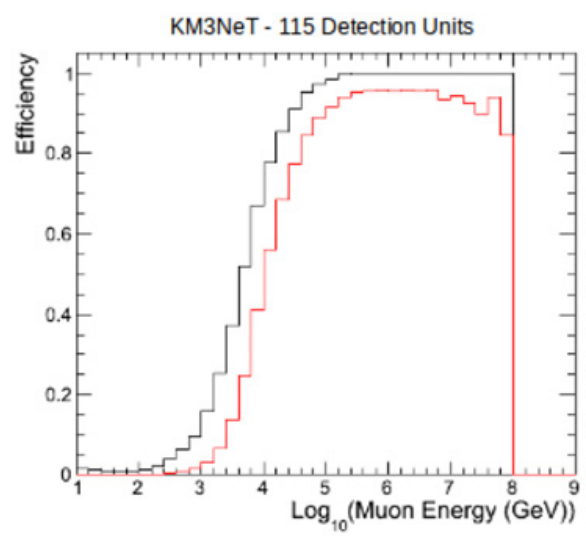

Figure 1. The efficiency of the reconstruction as a function of $\log _{10} E_{\mu}$. The black line corresponds to all events that cross the detector and the red line refers to events satisfying the selection criteria.

energy ranging from $10^{2} \mathrm{GeV}$ to $10^{8} \mathrm{GeV}$. The first event sample assumes that neutrinos are generated with an energy spectrum $E^{-1.4}$ while the second event sample corresponds to a neutrino energy spectrum of $E^{-2}$. A random optical background rate of $5 \mathrm{kHz}$ was assumed for each PMT, including dark current, ${ }^{40} \mathrm{~K}$ decays and bioluminescence. In addition to random coincidences, a rate of $500 \mathrm{~Hz}$ due to genuine coincidences from ${ }^{40} \mathrm{~K}$ decay has also taken into account.

\section{Track direction reconstruction}

The simulated events were reconstructed using the Chameleon reconstruction package [2]. This package consists of two main parts. The pattern recognition which includes algorithms for the selection and grouping of hits in track candidates and the fitting algorithm that uses a $\chi^{2}$ minimizer in order to reconstruct the direction of the track.

A quality selection process is applied: A minimum number of OMs with hits, and a minimum path inside the instrumented volume are required in order to get a reliable reconstruction of the track direction. The efficiency and the purity of the track direction reconstruction is reported in Figs. 1 and 2, respectively, where the black line corresponds to all events that cross the detector volume and the red line refers to events that survive from the quality selection process. The purity of the reconstruction refers to the ratio of the well reconstructed events (events for which the angle difference $(\Delta \Omega)$ between the simulated and the reconstructed muon track direction is less $1^{\circ}$ ) to all reconstructed events that survive from the quality selection process. A good purity $(\geq 80 \%)$ of the reconstructed events especially in high energies $\left(E_{\mu} \geq 10 \mathrm{TeV}\right)$ can be established, accompanied by an efficiency of $\simeq 55 \%$ for $E_{\mu} \geq 10 \mathrm{TeV}$ and $\geq 80 \%$ for $E_{\mu} \geq 40 \mathrm{TeV}$ rising with energy to approximately $\simeq 100 \%$ for $E_{\mu} \geq 100 \mathrm{TeV}$.

The median of the angle difference $(\Delta \Omega)$ between the simulated and the reconstructed track direction is shown in Fig. 3. The median is less than $0.5^{\circ}$ for $E_{\mu}>1 \mathrm{TeV}$ while it reaches $0.35^{\circ}$ for $E_{\mu}=10 \mathrm{TeV}$. A very good angular resolution is achieved in the high energy regime with the median of $\Delta \Omega=0.15^{\circ}$ for $E_{\mu} \simeq 3 \mathrm{PeV}$. For muon ener-gies above $10 \mathrm{PeV}$ the calculation of the median is domi-nated by limited statistics. 


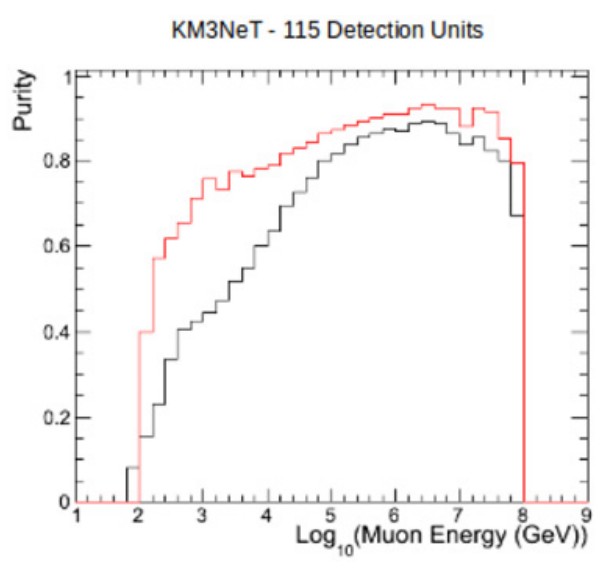

Figure 2. The purity of the reconstruction that is defined as the ratio of well reconstructed events $\left(\Delta \Omega \leq 1^{\circ}\right)$ to the number of reconstructed events as a function of $\log _{10} E_{\mu}$. The black line corresponds to all events that cross the detector and the red line refers to events satisfying the selection criteria.

KM3NeT - 115 Detection Units
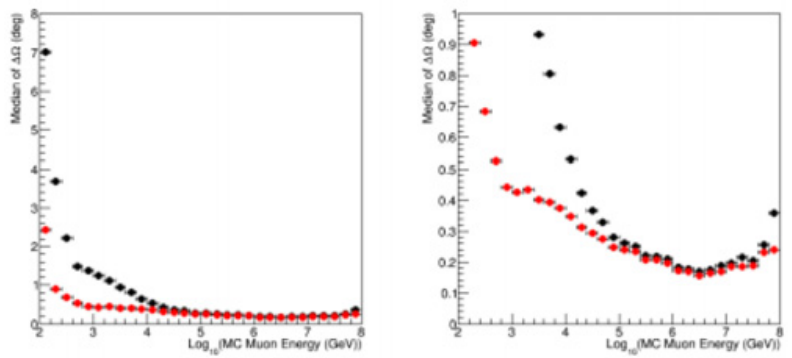

Figure 3. The median of the angle difference $(\Delta \Omega)$ between the simulated and the reconstructed muon track direction as a function of $\log _{10} E_{\mu}$. The right plot depicts the distribution of the median of this angle difference for $\Delta \Omega<1^{\circ}$ while the left plot contains all values of median with respect to $\log _{10} E_{\mu}$. The black dots correspond to all reconstructed events and the red dots refer to events satisfying the selection criteria.

\section{Energy estimation}

Muons lose energy via ionization and by stochastic processes, such as bremsstrahlung, pair production, and photonuclear interactions. The total average energy loss of the muon is:

$$
\frac{-d E}{d x}=a(E)+b(E) E_{\mu}
$$

where $a \simeq 0.274 \mathrm{GeV} \mathrm{m}^{-1}$ accounts for the energy loss due to ionization and $b \simeq 0.000349 \mathrm{~m}^{-1}$ is due to the stochastic energy loss. Muons with $E_{\mu}>1 \mathrm{TeV}$ lose energy stochastically, while for lower energies ionization dominates. We describe a method to derive muon (and neutrino) energy from the light collected along its passage through the detector volume.

When the neutrino interacts inside the instrumented volume, both photons from the hadronic shower and Cherenkov photons from the muon passage through sea water can be detected, leading to a reliable estimate of the neutrino energy. If the neutrino has interacted 
to muon far outside the instrumented volume, the majority of the light produced by the muon (and/or hadronic shower) never reaches the PMT sensors, leading to an underestimation of the neutrino energy. In this case the muon energy is used as a measure of the neutrino energy. In order to ensure that photons from the hadronic part can be detected, the neutrino energy is estimated for events with the reconstructed neutrino interaction vertex inside a cylindrical fiducial volume, which is slightly smaller than the detector volume. For events that cross the detector volume but the reconstructed neutrino interaction vertex lies outside the fiducial volume, only the muon energy is reconstructed. The MC muon energy that is considered for this study refers to the energy of the muon at the neutrino interaction vertex.

\subsection{Neural network}

Artificial neural networks (NNs) are gaining ground as a multipurpose, robust computational methodology which performs effectively in many analyses. The response of an artificial neural network is determined by the layout of the neurons, the weights of the inter-neuron connections and the neuron response function which describes the response of the neurons to the input variable. This analysis is based on the employment of a Multi-Layer Percepton (MLP) Neural Network which is part of the TMVA package included in ROOT [3]. The neurons in such neural network are organized in layers and only direct connections from a given layer to the following layer are allowed resulting in a less complex system. The first layer of a MLP is the input layer that hosts the neurons. Each neuron holds an input variable. The last layer is the output layer that holds the output variable, the neural network estimator. All intermediate layers are the hidden layers of the NN. A weight is associated to each directional connection between the output of one neuron and the input of another neuron. During the calculation of the input value to the response function of a neuron these weights are multiplied with the output values of all neurons connected to this given neuron [3]. The NN used for the current analysis consists of three layers. The first layer holds four input variables, the hidden layer consists of 10 nodes and the output layer returns the network result. The neuron activation function used was the hyperbolic tangent while the weights were adjusted with the use of the Broyden-Fletcher-Goldfarb-Shannon (BFGS) method [4].

\subsection{Description of the neural network input variables for the energy estimation}

Since the determination of neutrino and muon energy is based on the collection of light in PMTs, muons should have traveled an adequate distance inside the volume of the detector before an attempt to evaluate their energy is made. Furthermore, the optical modules (OMs) in the detector configuration are not homogeneously distributed in space. The distance of OMs on a string is less than half of the distance between neighboring strings. As a consequence the minimum expected path of a muon through the detector should differ with the reconstructed muon zenith angle. We define the minimum expected distance by the formula:

$$
\text { Minimum Expected Path }=0.5 * h+(R-0.5 * h) * \sin \left(\theta_{\text {rec }}\right)
$$

where: $\mathrm{h}$ is the string height, $\mathrm{R}$ is the detector radius and $\theta_{\text {rec }}$ the reconstructed muon angle $\left(\theta_{\text {rec }}=\pi-\theta_{\text {zenith }}\right)$.

According to this formula the "minimum expected path" for horizontal muons is the detector radius, while for vertical muons it is half of the height of the string. We calculate the distance between the first and the last PMT position along the track (the reconstructed track direction is not used in order to avoid bias due to poorly reconstructed events) and we 
accept events that fulfill the requirement:

$$
\frac{\text { Distance between PMTs }}{\text { Minimum Expected Path }}>0.5 \text {. }
$$

The quantities that have a strong dependence on the muon and neutrino energy and are used as input variables to feed the Neural Network are:

i. The number of OMs used in the reconstruction divided by the calculated muon length. A weight is used in order to also accept events with low energies that stop traveling inside the detector.

$$
\frac{\text { Number of } O M s}{\text { muon length }} * \frac{\text { muon length }}{\text { muon potential length }}
$$

ii. The number of PMTs that have L1 pulses (coincidence of two PMTs in the same OM in a time interval of $10 \mathrm{~ns}$ ) and were used in the reconstruction weighted according to the vertical distance from the track.

$$
\sum_{i=1}^{\text {Number of PMTs }} \frac{D_{i}}{D_{\max }}
$$

where $D_{i}$ is the vertical distance between the PMT and the reconstructed track and $D_{\max }=500 \mathrm{~m}$.

iii. The ratio of the total number of PMTs that have L1 pulses and were used in the reconstruction to the number of PMTs that could be hit according to the track and the PMT direction but have not recorded any pulses.

$$
\frac{\text { Number of PMTs with hits }}{\text { Number of PMTs with no hits }}
$$

iv. The Total Time over Threshold (ToT) of all PMTs used in the reconstruction. The Time over Threshold is used as a measure of charge in PMTs.

$$
\sum_{i=1}^{\text {Number of PMTs }} \text { ToT. }
$$

\subsection{Results on muon and neutrino energy reconstruction}

Once the weights of the inter-neuron connections of the NN have been produced, a different sample of $v_{\mu}$ events is used for the evaluation of the Neural Network and for the estimation of muon and neutrino energy. The input variables are created for this new sample and are inserted in the $\mathrm{NN}$ which returns the logarithm of the reconstructed energy in GeV.

The relation between the estimated and the MC energy for muons and neutrinos is presented in Figs. 4 and 5 respectively. The energy range of interest for this experiment refers to muon and neutrino energy above $1 \mathrm{TeV}$ and this is the energy range used in the figures above. An overestimation of the muon energy for $E_{\mu}<10 \mathrm{TeV}$ can be observed which recedes as the energy increases resulting to a linear correlation for $E_{\mu} \geq 10 \mathrm{TeV}$. This overestimation is expected as the track direction reconstruction applies a strict hit selection in order to reduce hits coming from background sources, reconstructing mainly events with $E_{\mu} \geq 10 \mathrm{TeV}$ (see Fig. 1). A very good correlation between the reconstructed and simulated energy can still be observed for $E_{v} \geq 10 \mathrm{TeV}$ while the deviation from the line for $E_{v}>600 \mathrm{TeV}$ is due to low statistics. 


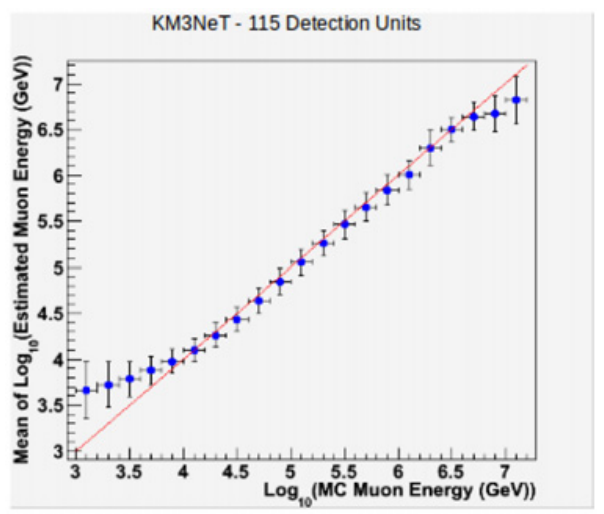

Figure 4. The mean (with RMS error) of the distribution of the logarithm of the reconstructed muon energy with respect to the logarithm of the MC muon energy.

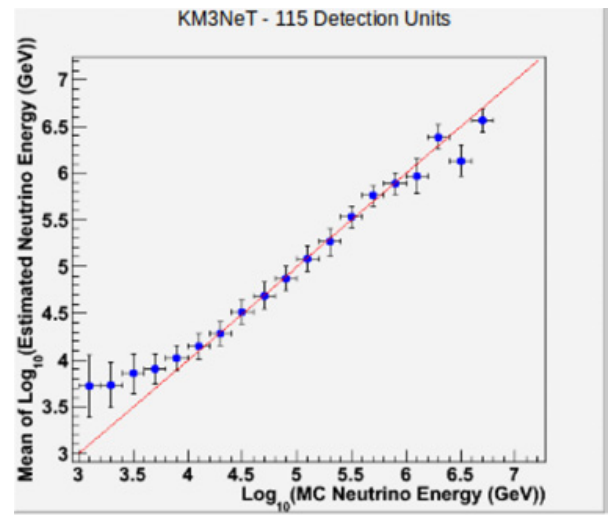

Figure 5. The mean (with RMS error) of the distribution of the logarithm of the reconstructed neutrino energy with respect to the logarithm of the MC neutrino energy for events with the reconstructed neutrino vertex inside the fiducial volume.

Figure 6 shows the distribution of $\log _{10}$ (Estimated Muon Energy/MC Muon Energy) for all events for which the energy was estimated $\left(100 \mathrm{GeV} \leq E_{\mu} \leq 20 \mathrm{PeV}\right)$. The distribution is fit with a Gaussian. An offset of the mean at 0.14 can be observed which depicts the overestimation of energy for $E_{\mu}<1 \mathrm{TeV}$ due to the fact that in these energies ionization dominates, while the $\sigma \simeq 0.32$. This overestimation of muon energy is absent at high muon energies $\left(E_{\mu} \geq 10 \mathrm{TeV}\right)$ for which a mean at $\simeq-0.03$ and a $\sigma \simeq 0.26$ is achieved (Fig. 7).

Figure 8 shows the distribution of $\log _{10}$ (Estimated Neutrino Energy/MC Neutrino Energy) for events with the reconstructed neutrino vertex inside the fiducial volume. The distribution is fit with a Gaussian. An offset of the mean at 0.10 can be observed which depicts the overestimation of energy for $E_{v}<1 \mathrm{TeV}$ due to the fact that in these energies ionization dominates, while the $\sigma \simeq 0.29$. This overestimation of neutrino energy is absent at high neutrino energies $\left(E_{v} \geq 10 \mathrm{TeV}\right.$ ) for which a mean at $\simeq 0.014$ and a $\sigma \simeq 0.26$ is achieved (Fig. 9).

The muon energy resolution for all events for which the energy was estimated and the neutrino energy resolution for events with the reconstructed neutrino vertex inside the fiducial volume for three different energy intervals is reported in Fig. 10. The energy intervals were 


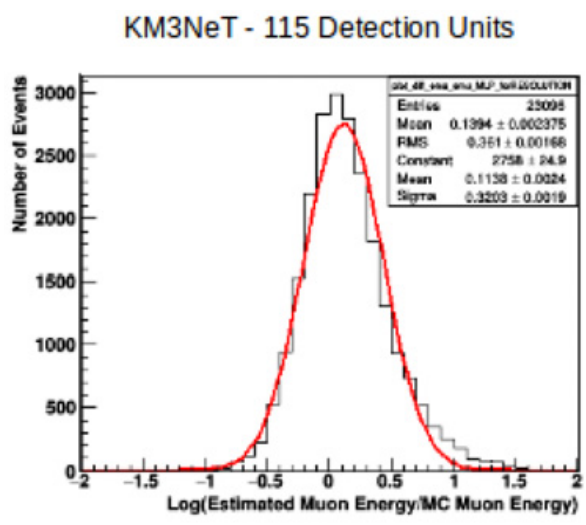

Figure 6. The distribution of $\log _{10}$ (Estimated Muon Energy/MC Muon Energy) for all events for which the energy was estimated.

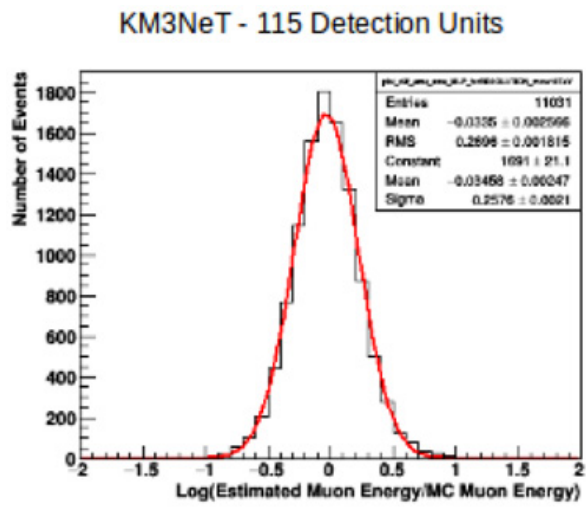

Figure 7. The distribution of $\log _{10}$ (Estimated Muon Energy/MC Muon Energy) for events with $E_{\mu} \geq 10 \mathrm{TeV}$ for which the energy was estimated.

KM3NeT - 115 Detection Units

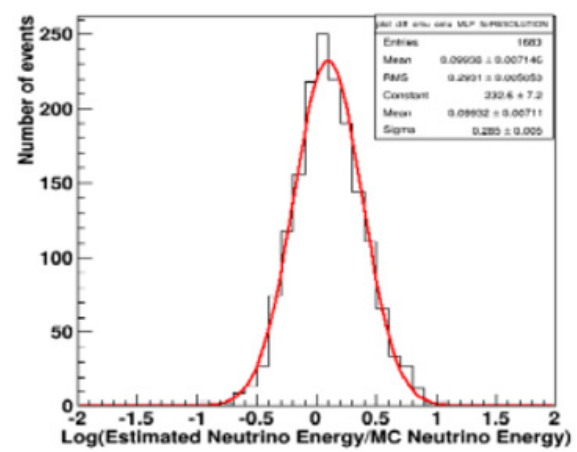

Figure 8. The distribution of $\log _{10}$ (Estimated Neutrino Energy/MC Neutrino Energy) for events with the reconstructed neutrino vertex inside the fiducial volume. 


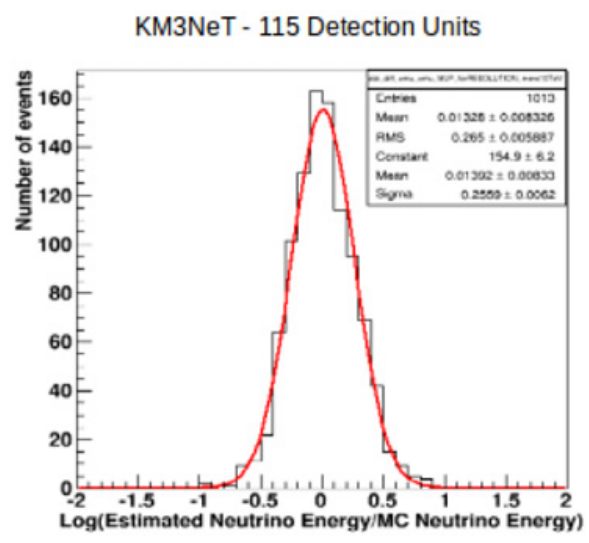

Figure 9. The distribution of $\log _{10}$ (Estimated Neutrino Energy/MC Neutrino Energy) for events with $E_{\mu} \geq 10 \mathrm{TeV}$ for which the reconstructed neutrino vertex is inside the fiducial volume.

\section{Energy Resolution}

\begin{tabular}{|c|c|c|c|}
\hline & $1 \mathrm{TeV} \leq E_{\mu}<10 \mathrm{TeV}$ & $10 \mathrm{TeV} \leq E_{\mu}<30 \mathrm{TeV}$ & $30 \mathrm{TeV} \leq E_{\mu}<20 \mathrm{PeV}$ \\
\hline $\begin{array}{c}\text { Muons crossing the } \\
\text { detector volume }\end{array}$ & 0.29 in $\log _{10} E_{\mu}$ & 0.25 in $\log _{10} E_{\mu}$ & 0.26 in $\log _{10} E_{\mu}$ \\
\hline $\begin{array}{c}\text { Events with the } \\
\text { reconstructed neutrino } \\
\text { vertex inside a fiducial } \\
\text { volume }\end{array}$ & 0.27 in $\log _{10} E_{v}$ & 0.26 in $\log _{10} E_{v}$ & 0.25 in $\log _{10} E_{v}$ \\
\hline
\end{tabular}

Figure 10. The energy resolution for three different energy intervals.

chosen in order to have comparable number of events in each energy range. The energy resolution is determined by the standard deviation of the distribution of $\log _{10}$ (Estimated Muon Energy/MC Muon Energy) and $\log _{10}$ (Estimated Neutrino Energy/MC Neutrino Energy) respectively. The muon energy resolution for $1 \mathrm{TeV} \leq E_{\mu}<10 \mathrm{TeV}$ is 0.29 and it decreases to 0.25 for $10 \mathrm{TeV} \leq E_{\mu}<30 \mathrm{TeV}$ and 0.26 for $30 \mathrm{TeV} \leq E_{\mu}<20 \mathrm{PeV}$. The neutrino energy resolution corresponds to 0.27 for $1 \mathrm{TeV} \leq E_{v}<10 \mathrm{TeV}$ and it decreases with the rising of the neutrino energy to 0.26 for $10 \mathrm{TeV} \leq E_{v}<30 \mathrm{TeV}$ and 0.25 for $30 \mathrm{TeV}$ $\leq E_{v}<20 \mathrm{PeV}$.

\section{Conclusions}

A new method for the muon and neutrino energy estimation using a Multi-Layer Percepton Neural Network with appropriate input variables was presented. The results achieved lead to an energy resolution $\simeq 0.26$ in $\log _{10} E_{\mu}$ for muon energy at $10 \mathrm{TeV} \leq E_{\mu} \leq 20 \mathrm{PeV}$, while for muons over all energies $\left(100 \mathrm{GeV} \leq E_{\mu} \leq 20 \mathrm{PeV}\right)$ the energy resolution is $\simeq 0.32$ in $\log _{10} E_{\mu}$. For the events with the neutrino vertex inside the fiducial volume the energy resolution is $\simeq 0.26$ in $\log _{10} E_{v}$ for neutrino energy at $10 \mathrm{TeV} \leq E_{v} \leq 20 \mathrm{PeV}$, while for neutrinos over all energies $\left(100 \mathrm{GeV} \leq E_{v} \leq 20 \mathrm{PeV}\right)$ the energy resolution is $\simeq 0.29$ in $\log _{10} E_{v}$.

The presented work has been partially financed by the European Social Fund (NSRF 2007-2013) under the Thalis initiative GRBNeT project-MIS 360381. I would like to thank Katerina Tzamariudaki and 
Christos Markou for their invaluable assistance during the supervision of the present work. I also owe many thanks to Konstantia Balasi for converting the initial event samples to the appropriate format used by the chameleon package and to Kostantinos Pikounis for providing his help whenever it was necessary.

\section{References}

[1] V. Agrawal, T. K. Gaisser, P. Lipari, T. Satnev, Phys Rev D. 53.1314 v1, 18 (1995)

[2] D. Lenis, G. Stavropoulos, astro-ph.IM, v1, 37 (2012)

[3] A. Hoecker, P. Speckmayer, J. Stelzer, J. Therhaag, E. von Toerne, H. Voss, TMVAUsersGuide, 152 (2013)

[4] C. G. Broyden, The Convergence of a Class of Double-rank Minimization Algorithms, J.Inst. of Math. and App. 6, 76 (1970); R. Fletcher, A New Approach to Variable Metric Algorithms, Computer J. 13, 317 (1970); D. Goldfarb, A Family of Variable Metric Updates Derived by Variational Means, Math. Comp. 24, 23 (1970); D.F. Shannon, Conditioning of Quasi-Newton Methods for Function Minimization, Math. Comp. 24, 647 (1970) 